\title{
Efficacy and Safety of Ledispavir/Sofosbuvir with or without Ribavirin in patients with Decompensated Liver Cirrhosis and Hepatitis C Infection: a Cohort Study
}

\author{
Liliana Simona Gheorghe ${ }^{1}$, Carmen Monica Preda ${ }^{1}$, Laura Iliescu ${ }^{2}$, Doina Istratescu ${ }^{3}$, Andreea Elena Chifulescu ${ }^{3}$, \\ Corina Silvia Pop ${ }^{4}$, Anca Trifan ${ }^{5}$, Carol Stanciu ${ }^{5}$, Mircea Diculescu ${ }^{1}$, Theodor Voiosu ${ }^{6}$, Cristian Baicus ${ }^{6}$, Letitia Tugui ${ }^{3}$, \\ Speranta Iacob ${ }^{1}$, Cristian Tieranu ${ }^{7}$, Corina Meianu ${ }^{1}$, Mircea Manuc ${ }^{1}$
}

1) Carol Davila University

of Medicine and Pharmacy, Gastroenterol and Hepatol Depart, Clinic Fundeni Institute, Bucharest; 2) Carol Davila University of Medicine and Pharmacy, Internal

Medicine Depart, Clinic Fundeni Institute, Bucharest; 3) Gastroenterol and Hepatol Depart, Clinic Fundeni Institute, Bucharest; 4) Carol Davila University of Medicine and Pharmacy, Internal Medicine Depart, Emergency Universitary Hospital, Bucharest; 5) Gr. T. Popa University of Medicine and Pharmacy, Gastroenterol and Hepatol Depart, Gastroenterol and Hepatol Institute, Iasi; 6) Carol Davila University of Medicine and Pharmacy, Internal Medicine Depart,

Colentina Hospital, Bucharest

7) Carol Davila University of Medicine and Pharmacy, Gastroenterol and Hepatol Depart, Elias Emergency Hospital, Bucharest, Romania

Address for correspondence: Carmen Monica Preda Carol Davila University of Medicine and Pharmacy, Gastroenterol and Hepatol Department, Clinic Fundeni Institute, 258 Sos. Fundeni, Bucharest, Romania carmenmonica.preda@gmail.com

Received: 26.04 .2020 Accepted: 07.07.2020

\section{ABSTRACT}

Background \& Aims: Ledipasvir/Sofosbuvir (LDV/SOF) with or without Ribavirin (RBV) has shown good results in terms of efficacy and safety in clinical trials in advanced liver cirrhosis, but real-life data are still needed in order to confirm this profile. We investigated the efficacy and safety of LDV/SOF in a large Romanian population with liver cirrhosis and genotype $1 \mathrm{~b}$ hepatitis $\mathrm{C}$ virus (HCV).

Methods: We analyzed a multicentric retrospective cohort enrolling 349 patients with decompensated liver cirrhosis due to HCV who received LDV/SOF \pm RBV 12/24 weeks (301/48). Patients were included between 2017-2018, all with genotype 1b. Main inclusion criteria were liver cirrhosis and detectable HCV RNA. The cases were followed-up monthly during therapy and 12 weeks after the end of therapy.

Results: The cohort included $60 \%$ females with a median age of $61,16 \%$ interferon (IFN) pre-treated, 53\% with comorbidities, $40 / 53 / 7 \%$ with Child Pugh A/B/C, $4 \%$ with virus B co-infection and $8 \%$ with previously treated hepatocellular carcinoma. Mean initial MELD score was $11.92(6.82 \div 24.5)$. Six patients were lost during follow-up. Sustained virologic response (SVR) in intention-to-treat was reported in $85.1 \%$. Predictive factors of SVR in decompensated cirrhosis were female gender $(\mathrm{p}=0.01)$, advanced age $(\mathrm{p}<0.001)$, lower bilirubin levels $(\mathrm{p}=0.002)$ and lower CTP score $(\mathrm{p}=0.02)$. In patients with CTP score $B$ or $\mathrm{Cl}$ low bilirubin levels $(\mathrm{p}=0.003)$, low INR $(p<0.001)$, increased platelet count $(\mathrm{p}=0.04)$, low CTP score $(\mathrm{p}<0.001)$, lack of encephalopathy $(\mathrm{p}=0.02)$, serum albumin $>3.5 \mathrm{~g} / \mathrm{dl}(\mathrm{p}=0.002)$ predicted improvement of liver function. Serious adverse events were reported in $16 / 349$ (4.6\%), most of them due to severe liver decompensation $(9 / 16)$.

Conclusions: $\mathrm{LDV} / \mathrm{SOF} \pm \mathrm{RBV}$ proved to be highly efficient in our difficult to treat population with $85.1 \% \mathrm{SVR}$.

Key words: ledipasvir/sofosbuvir - ribavirin - liver cirrhosis - hepatitis C virus - direct acting antiviral agents.

Abbreviations: ALT: alanine aminotransferase; AST: aspartate aminotransferase; CTP score: Child-TurcottePugh score; DAA: direct-antiviral agent; EASL: European Association for the Study of the Liver; EOT: end of treatment; HBV: hepatitis B virus; HCC: hepatocellular carcinoma; HCV: hepatitis C virus; IFN: interferon; INR: international normalized ratio; ITT: intention-to-treat; LDV/SOF: ledipasvir-sofosbuvir; MELD: Model for End-stage Liver Disease; PCR: polymerase chain reaction; RBV: ribavirin; SVR: sustained virologic response.

\section{INTRODUCTION}

Hepatitis $\mathrm{C}$ virus (HCV) is an epidemic problem worldwide, affecting approximately 71 million people $[1,2]$. In Romania, the estimated prevalence of chronic $\mathrm{HCV}$ according to data published in 2010 was approximately $4 \%$ [3], but it is decreasing now due to reimbursed direct directantiviral agents (DAAs), received by approximately 35,000 patients with more than a $96 \%$ sustained virologic response (SVR) rate [4-6]. In Romania, genotype $1 \mathrm{~b}$ is almost exclusively present among the infected patients [7].

Ledipasvir/Sofosbuvir (LDV/SOF) was approved as the treatment for genotype 1 chronic HCV infection in October 2014. Efficacy and safety of LDV/SOF with or without Ribavirin (RBV) in compensated liver cirrhosis and genotype $1 \mathrm{HCV}$ infection have been demonstrated in clinical trials, with a SVR rate of $96 \%$ [8]. Real-world data that included around 5,070 patients, reported a SVR rate of $93-98 \%$, with very good tolerance [9-15]. LDV/SOF was tested in decompensated liver cirrhosis in two major clinical trials: SOLAR-1 and SOLAR-2, 
which reported a SVR of $86 \%$ for a 12 week course of LDV/ SOF and RBV [16, 17]. Real-life data reported a lower SVR of $78-83 \%$, but also a good tolerance [18-21]. Model for end-stage liver disease (MELD) scores improved in treated patients (mean change 0.85) [18]. Patients with initial serum albumin $<35 \mathrm{~g} / \mathrm{L}$, aged $>65$ or with low baseline serum sodium concentrations $(<135 \mathrm{mmol} / \mathrm{L})$, with ascites or encephalopathy, alanine aminotransferase (ALT) $<60 \mathrm{U} / \mathrm{L}$, and body mass index $>25 \mathrm{~kg} / \mathrm{m}^{2}$ were least likely to benefit from therapy $[18,22]$.

The aim of the present study was to investigate the efficacy and safety of LDV/SOF in a real-world large Romanian population with liver cirrhosis caused by genotype $1 \mathrm{~b} \mathrm{HCV}$.

\section{METHODS}

Out of the 772 patients with advanced HCV liver cirrhosis who received reimbursed DAAs therapy with LDV/SOF with or without RBV for 12-24 weeks during 2017-2018 in Romania, we analyzed a multicentric retrospective cohort enrolling 349 patients who started the therapy between September 2017 and July 2018 in the Gastroenterology Department and Internal Medicine Department of the Fundeni Clinic Institute, Bucharest, the Gastroenterology and Hepatology Institute Iasi, the Internal Medicine Department of the Emergency University Hospital Bucharest and the Gastroenterology Department of Elias Emergency Hospital, Bucharest, all with genotype $1 \mathrm{~b}$ infection. The inclusion criteria were decompensated liver cirrhosis, detectable HCV-RNA viral load in serum, no significant ethanol consumption in the last 3 months. All the potential drug-drug interactions were checked, and concomitant therapy was administered only if potential interactions were excluded.

The diagnosis of cirrhosis was based on a liver biopsy detecting fibrosis corresponding to Metavir F4 score. In the absence of the liver biopsy, the presence of cirrhosis was proven by a median liver stiffness measurement $\geq 12.5 \mathrm{kPa}$ at transient elastography (Fibroscan), advanced fibrosis (F4) confirmed by Fibromax testing or signs of portal hypertension (esophageal or gastric varices, portal gastropathy). The definition of decompensated cirrhosis was the presence of prior or current variceal hemorrhage, ascites, hepatic encephalopathy or hepatic hydrothorax.

According to the therapeutic guidelines for the reimbursement of DAAs therapy, patients co-infected with hepatitis B virus (HBV) received concomitant anti-HBV therapy with Entecavir during their anti-HCV therapy. Those with hepatocellular carcinoma (HCC) were treated if they had an absence of HCC relapse 3 months after their last session of therapy (surgery, radiofrequency ablation or transarterial chemoembolization).

Only serious adverse events leading to discontinuation of therapy were reported.

The study was approved by the National Ethics Committee of Medicines and Medical Devices (No 27SNI/October 10, 2016). All patients signed a written informed consent before entering the study.

Main outcome measures: efficacy of the DAAs therapy was assessed by the percentage of patients achieving SVR (HCV RNA undetectable) 12 weeks post-treatment (SVR12). To evaluate the liver function, the MELD score and the Child
Turcotte-Pugh (CTP) score were calculated at the start of DAAs therapy, at the end of treatment (EOT) and at the SVR12.

The recorded patient data for analysis were: age, gender, prior antiviral therapy (and the patients' status: non-responder or relapser), presence of ascites, presence of encephalopathy and the degree of encephalopathy, history of variceal bleeding, presence of esophageal varices, presence of significant comorbidities, use of concomitant medications. Laboratory data were recorded 3 months before starting the antiviral therapy, at EOT and 12 weeks after the EOT. Parameters recorded included: platelet count, international normalized ratio (INR), total bilirubin, aspartate aminotransferase (AST), ALT, glucose level, albumin, HBs antigen, alpha-fetoprotein, creatinine and estimated clearance of creatinine. All patients who completed the antiviral therapy had their HCV-RNA viral load determined at the end of therapy and at 12 weeks after the end of therapy. HCV-RNA levels were assessed by quantitative polymerase chain reaction (PCR) assays: COBAS TaqMan HCV v2.0 (Roche Molecular Diagnostics, Pleasanton, CA, USA).

The patients were enrolled for treatment with LDV/SOF with or without RBV according to the therapeutic guidelines of the EASL and the manufacturer's recommendations [7].

A total of 301 patients received one tablet of LDV/SOF (90/400 mg per tablet) with or without RBV (starting dose of $600 \mathrm{mg}$, which can be titrated up to a maximum of $1,000 / 1,200$ $\mathrm{mg}$ ) for 12 weeks. Forty-eight cases considered ineligible for or intolerant to RBV were treated with LDV/SOF (90/400 mg) daily for a total of 24 weeks. These patients were with moderatesevere anemia, hemoglobinopathies (i.e. thalassemia major), decompensated heart failure or with poor tolerance to RBV on previous antiviral treatment.

All analysis was conducted in intention-to-treat (ITT). Results were summarized as median and range for nonnormally distributed scales or ordinal variables or as numbers and percentages for categorical variables. We looked for differences concerning the independent variables by the outcome in bivariate analysis (Mann-Whitney U test or Fisher's exact test, depending on variables). A logistic regression model was computed, and all independent variables associated with $\mathrm{p}<0.10$ with the dependent variable in bivariate analysis were introduced in a stepwise manner. The model with the independent variables retained by both the forward and the backward stepwise method was kept. A two-sided $p$ value of $<0.05$ was noted as statistically significant. Data analyses were performed with statistical software (Stata 11 from StataCorp LP, College Station, TX, USA, and SPSS version 20.0 from IBM Corporation, Armonk, NY, USA).

\section{RESULTS}

There were 349 patients with decompensated cirrhosis included in the cohort. Six patients were lost during follow-up and in $16(4.6 \%)$ the treatment was interrupted due to adverse events: nine developed worsening hepatic function $(2.6 \%)$, four experienced cardiovascular adverse events, one developed severe allergy and two had severe bacterial infections (Fig. 1). Baseline characteristics of patients with decompensated cirrhosis treated with LDV/SOF with or without RBV are shown in Table I. 


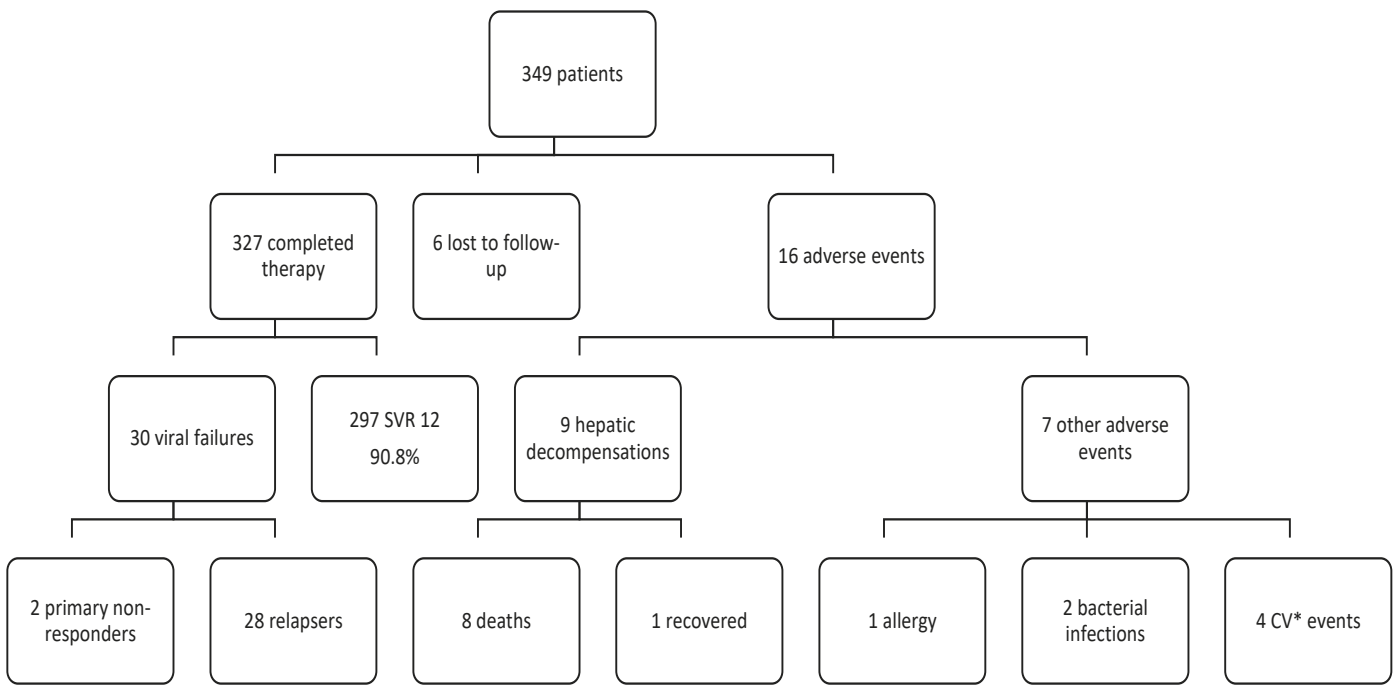

Fig. 1. The study flowchart. CV: cardiovascular

Table I. Baseline characteristics of patients with decompensated cirrhosis treated with LDV/SOF with or without RBV

\begin{tabular}{|c|c|}
\hline Decompensated liver cirrhosis & 349 \\
\hline $\operatorname{Age}^{\star}$ (years) & $61(35-83)$ \\
\hline Gender male (\%) & 41.3 \\
\hline Co-morbidities (\%) & 53 \\
\hline IFN Pre-treated (\%) & 15.8 \\
\hline HBs-Antigen positive (\%) & 4 \\
\hline Treated HCC & 8 \\
\hline $\operatorname{AST}^{*}(\mathrm{IU} / \mathrm{ml})$ & $69(20-396)$ \\
\hline $\operatorname{ALT}^{*}(\mathrm{IU} / \mathrm{ml})$ & $58(15-497)$ \\
\hline HCV-RNA* (IU/l) & $247,000(55-7,276,049)$ \\
\hline Total bilirubin* (mg/dl) & $1.8(0.3-11)$ \\
\hline MELD* score & $11.92(6.82-24.5)$ \\
\hline CPT score $(\mathrm{A} / \mathrm{B} / \mathrm{C})(\%)$ & $40 / 53 / 7$ \\
\hline
\end{tabular}

${ }^{*}$ median (minim-maxim); AST: aspartate aminotransferase; ALT: alanine aminotransferase; CTP: Child-Turcotte-Pugh; HCC: hepatocellular carcinoma; IFN: interferon; LDV/SOF: ledispavir/sofosbuvir; MELD: Model for End-stage Liver Disease; RBV: ribavirin; SVR: sustained virologic response

Briefly, this group consisted of $58.7 \%$ females; patients had a mean age of 61 years (35-83) and $16 \%$ had failed prior interferon-based therapies $53 \%$ of patients had comorbidities, most frequently being cardiovascular disease: $24.6 \%$ hypertension, $8 \%$ ischemic heart disease, $7 \%$ chronic heart failure, $3 \%$ atrial fibrillation, $2.6 \%$ had a history of stroke and $2 \%$ various valvular disorders. The prevalence of type 2 diabetes in our cohort was $23.5 \%$. Other comorbidities were portal vein thrombosis: $3 \%$, different types of anemia: $3 \%$, different thyroid disorders: $3 \%$, chronic obstructive pulmonary disease: $2.3 \%$, colonic cancer operated $2 \%$, peptic ulcer disease $1.7 \%$, symptomatic cryoglobulinemia $1.4 \%$.

Sustained virologic response rate by ITT analysis was reported in $85.1 \%(297 / 349)$, and per protocol was $90.8 \%$ (297/327). Sustained virologic response rate by ITT was $87.8 \%$ in patients with CTP A score at therapy initiation, $84 \%$ in patients with CTP B score and decreased further to $78.3 \%$ in those with CTP C ( $\mathrm{p}=0.02)$.

In univariate analysis the predictive factors for SVR12 were in our cohort: female gender $(\mathrm{p}=0.01)$, advanced age $(\mathrm{p}<0.001)$, lower bilirubin levels $(\mathrm{p}=0.002)$ and lower CTP score $(\mathrm{p}=0.02)$ (Table II) (univariate analysis). The parameters that predicted SVR in a multivariable analysis were an age of more than 60 years (OR: 3.93; 95\%CI: 2.03-7.6) and total bilirubin level below $2 \mathrm{mg} / \mathrm{dl}$ (OR: 2.98; 95\%CI: 1.13- 3.84).

Table II. Predictive factors of SVR rate to in patients with decompensated liver cirrhosis (univariate analysis) by intention to treat

\begin{tabular}{lccc}
\hline Parameter & Responders & Non-responders & p-value \\
\hline Age $^{*}$ (years) & $61(35-83)$ & $56(37-72)$ & $<0.001$ \\
Male gender (\%) & 38.4 & 57.7 & 0.014 \\
Co-morbidities (\%) & 52.5 & 55.8 & 0.764 \\
CTP* score & $7(5-11)$ & $8(5-12)$ & 0.02 \\
HCV-RNA $^{*}(\mathrm{IU} / \mathrm{l})$ & 276000 & 167000 & 0.311 \\
& $(55-7276049)$ & $(11400-1970000)$ & \\
Total bilirubin* mg/dl & $1.5(0.3-9)$ & $2.2(0.4-11)$ & 0.002 \\
MELD ${ }^{*}$ score & 10.48 & 12.13 & 0.148 \\
& $(6.14-19.71)$ & $(6.26-24.5)$ & \\
Ascites at baseline (\%) & 42.8 & 42.3 & 1.000 \\
History of Variceal & 12.8 & 17.3 & 0.381 \\
bleeding (\%) & & & \\
\hline
\end{tabular}

For abbreviations see Table I

Three hundred and one cases received LDV/SOF/RBV for 12 weeks and 48 were treated with LDV/SOF for 24 weeks. Their baseline characteristics and response to therapy are described in Table III. The SVR rate did not significantly differ between the 2 arms of treatment: $84.7 \%$ for LDV/SOF/RBV and $87.5 \%$ for LDV/SOF ( $\mathrm{p}=0.83$ ). The proportion of RBV-induced anemia was $18.9 \%$. A percentage of 53.8 of the individuals treated with LDV/SOF/RBV improved their liver condition at SVR, compared with $58.3 \%$ of patients that received LDV/SOF $(p=0.104)$. Regarding the rate of adverse events occurrence, this was the same in the both groups: $4 \%$. 
Table III. Baseline characteristics and response to therapy in patients with compensated cirrhosis and HCV infection treated with SOF/LDV/ RBV versus SOF/LDV

\begin{tabular}{lccc}
\hline & $\begin{array}{c}\text { SOF/LDV/RBV } \\
12 \text { weeks }(\mathrm{n}=301)\end{array}$ & $\begin{array}{c}\text { SOF/LDV } \\
24 \text { weeks }(\mathrm{n}=48)\end{array}$ & p-value \\
\hline Male gender (\%) & 42.5 & 33.3 & 0.270 \\
Age* (years) & $61(35 \div 83)$ & $61(37 \div 80)$ & 0.34 \\
SVR12 (\%) & 84.7 & 87.5 & 0.827 \\
Co-morbidities (\%) & 49.5 & 75 & 0.001 \\
MELD & 10.63 & 11.13 & 0.022 \\
& $(6.14-24.5)$ & $(6.80-16.58)$ & \\
CTP score & $7(5 \div 12)$ & $6(5 \div 11)$ & 0.49 \\
\hline
\end{tabular}

For abbreviations see Table I

MELD score at EOT decreased to 10.91 (6.06-23.12) and dropped further to $9.78(6-19.84)$ at 3 months' interval (Fig. 2). At 12 weeks after the EOT, 38\% of cases maintained their liver function (also the CTP score) while $56 \%$ had improved liver function and $6 \%$ showed worsening liver function tests. From the 210 patients with CTP score $\mathrm{B}$ or $\mathrm{C}$ at therapy initiation, 97 improved to CTP score A (46.2\%). Seven percent of the included patients died during the follow-up period of 12 weeks after the EOT.

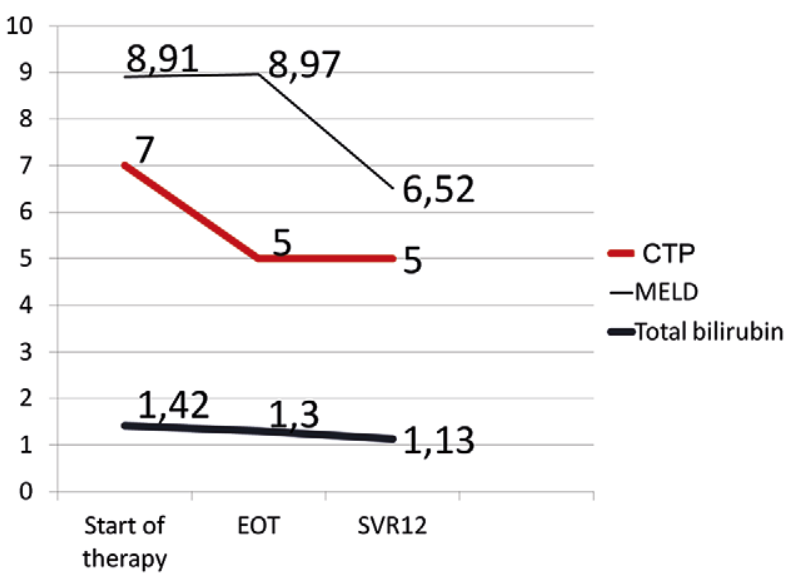

Fig. 2. Evolution of total bilirubin, MELD score and CTP (median) during DAAs therapy and at 12 weeks after the EOT.

At univariate analysis, we searched for predictors associated with improvements in decompensated cirrhosis after LDV/SOF with or without RBV therapy: lower levels of total bilirubin $(p=0.03)$, lower INR $(p<0.001)$, increased platelet count $(\mathrm{p}=0.04)$, low CTP score $(\mathrm{p}<0.001)$, lack of encephalopathy in patient's history $(\mathrm{p}=0.02)$, serum albumin $>3.5 \mathrm{~g} / \mathrm{dl}(\mathrm{p}$ 0.002) (Table IV). This was confirmed by the multivariable analysis (logistic regression): higher CTP score has an OR of 0.585 for improvement (95\%CI: 0.441-0.777; $\mathrm{p}<0.001$ ), lack of encephalopathy has 4.354 OR for improvement of liver function (95\%CI: 1.524-12.442; $\mathrm{p}=0.006$ ), serum albumin $>3.5 \mathrm{~g} / \mathrm{dl}$ has 3.317 OR for improvement (95\%CI: 1.317-8.357; $\mathrm{p}=0.01)$.

Serious adverse events were reported in 16/349 (4.6\%), most of them due to severe liver decompensation (9/16). The occurrence of adverse events was more frequent in patients with a more severe liver disease: increased CTP score $(\mathrm{p}<0.001)$, higher MELD scores $(\mathrm{p}=0.008)$. The subjects who developed severe adverse events had a median MELD score of 12.3 (8.83-21.7) and a median CTP score of 9 (7-12), compared to a median MELD score of $10.56(6.14-24.5)$ and a CTP score of $7(5 \div 11)$ in patients who did not experience severe adverse events. At multivariable analysis, only the CTP score was confirmed as a predictor for adverse events occurrence, with an OR of 1.678 (95\%CI: 1.205-2.336). Regarding the 4 cardiovascular events, there were 2 myocardial infarctions, 1 case of worsened heart failure and 1 ischemic stroke, resulting in 2 deaths (one patient with severe myocardial infarction and another one with stroke), probably not related to antiviral therapy or to drug-drug interactions.

Table IV. Predictive factors of improvement of Child score B/C to Child score $\mathrm{A}$ in patients with decompensated cirrhosis and $\mathrm{HCV}$ infection treated with LDV/SOF \pm RBV

\begin{tabular}{|c|c|c|c|}
\hline & $\begin{array}{c}\text { Patients with } \\
\text { CTP score B/C at } \\
\text { baseline and CTP } \\
\text { score A at SVR12 } \\
\mathrm{N}=97\end{array}$ & $\begin{array}{c}\text { Patients with } \\
\text { CTP score B/C at } \\
\text { baseline and CTP } \\
\text { score B/C at SVR12 } \\
\mathrm{N}=113\end{array}$ & $\mathrm{p}$-value \\
\hline Male gender (\%) & 42.3 & 38.9 & 0.673 \\
\hline Age $^{\star}$ (years) & $61(41-83)$ & $59(37-81)$ & 0.281 \\
\hline Co-morbidities (\%) & 52.6 & 47.8 & 0.580 \\
\hline Platelets $^{*}(x 109 / \mathrm{L})$ & $92(29-491)$ & $74(10-255)$ & 0.045 \\
\hline $\mathrm{INR}^{*}$ & $1.29(0.91-1.85)$ & $1.45(0.94-2.89)$ & $<0.001$ \\
\hline Total bilirubin* & $2.13(0.50-9)$ & $2.55(0.40-11)$ & 0.03 \\
\hline MELD $^{*}$ & $11.38(6.77-19.71)$ & $12.03(6.84-24.5)$ & 0.117 \\
\hline $\begin{array}{l}\text { No ascites at } \\
\text { baseline (\%) }\end{array}$ & 60.8 & 50.4 & 0.164 \\
\hline $\begin{array}{l}\text { History of variceal } \\
\text { bleeding (\%) }\end{array}$ & 10.3 & 15.9 & 0.309 \\
\hline $\mathrm{CTP}^{*}$ score & $7(7-10)$ & $8(7-12)$ & $<0.001$ \\
\hline $\begin{array}{l}\text { No history } \\
\text { of hepatic } \\
\text { enphalopathy (\%) }\end{array}$ & 93.8 & 83.2 & 0.019 \\
\hline $\begin{array}{l}\text { Serum albumin } \\
<3.5 \mathrm{~g} / \mathrm{dl}(\%)\end{array}$ & 75.3 & 91.2 & 0.002 \\
\hline ALT <60 IU/L (\%) & 48.5 & 55.8 & 0.333 \\
\hline
\end{tabular}

For abbreviations see Table I

\section{DISCUSSION}

The Food and Drug Administration approval in 2014 for $\mathrm{LDV} / \mathrm{SOF}$ in the treatment of chronic HCV infection has brought to the whole world an extremely efficient and well tolerated variant of DAAs treatment. Phase 3 registration studies estimated an SVR12 rate of 95\% in naive genotype 1 patients and $94 \%$ in experimented cases $[23,24]$. These registration studies included a relatively low number of individuals with compensated cirrhosis.

Our study reports a SVR12 per protocol of $90.8 \%$ in decompensated cirrhosis, which is very similar to the data resulting from earlier clinical trials: $86-87 \%$ in SOLAR-1 and SOLAR-2 $[16,17,28]$. Other real-life data report a SVR12 of $85-$ $91 \%$ in patients with decompensated cirrhosis $[14,18,28,29]$. 
We found two predictive factors for SVR in our cases with decompensated cirrhosis: advanced age (OR:0.949; 95\%CI: 0.919-0.980) and lower bilirubin levels (OR:1.310; 95\%CI: 1.126-1.532), similar to the findings of Lim JK et al [14] and Terrault et al. [27]. They reported two parameters associated with low SVR in their cohorts: albumin $<3.5 \mathrm{~g} / \mathrm{dl}$ and total bilirubin $>1.2 \mathrm{mg} / \mathrm{dl}[14,27]$.

No other clinical trial report or real-life study to date has found that male sex could be a predictive factor for nonresponse as we found in univariate analysis, but Lim JK et al [14] reported that a trend towards lower SVR was observed among males (OR: 0.49, 95\%CI: 0.20-1.18).

Data regarding safety in our cohort of patients treated with LDV/SOF with or without RBV were very good, with only 16 serious adverse events recorded in 349 patients (4.6\%). These data are similar with those reported by other authors [9-11, $13,14,25,26]$.

Regarding liver condition improvement in decompensated cirrhosis treated with DAAs, we identified a low CTP score, lack of encephalopathy, serum albumin $>3.5 \mathrm{~g} / \mathrm{dl}$ as predictors of improved liver function. Other authors found that body mass index $\leq 25 \mathrm{~kg} / \mathrm{m}^{2}$, the absence of hepatic encephalopathy and of ascites, albumin $>3.5 \mathrm{~g} / \mathrm{dl}$, and ALT $\geq 60 \mathrm{U} / \mathrm{L}$ were good predictors for achieving an improvement of CTP score to A [22]. A relatively preserved liver function predicted an improvement in the liver condition of the patients included in our study, due to the fact that a relatively small number of cases with MELD between 15 and 20 points were treated with LDV/SOF ( 25 out of 349 , that is $7.2 \%$ ). Only 3 individuals had MELD greater than 20 when included in treatment, and their evolution was very serious, with death recorded within the first 2 months of DAAs treatment due to hepatic decompensation and sepsis.

An important finding in our cohort is that there was no significant difference in SVR12 between cases treated with LDV/ SOF/RBV 12 weeks or 24 weeks of LDV/SOF. It is important to mention that the baseline characteristics of the cases with 12 weeks therapy duration versus 24 weeks are similar, with one exception: proportion of comorbidities is significantly increased in those that received 24 weeks of LDV/SOF: $75 \%$ versus $50 \%$. Other data from the literature confirm our findings [14], but there are authors that reported that SVR12 rates might be lower in patients with cirrhosis treated for 12 weeks (94 vs 99\%) [13].

Our study has several strengths: it represents a relatively large and homogenous cohort of patients with decompensated liver cirrhosis, HCV infection, all genotype $1 \mathrm{~b}$. This cohort included patients that are not represented in clinical trials: with HBV co-infection, with prior history of treated HCC.

The most important limitation of our study, inherent to all real-world observations, is that adverse events may be under-reported, because only those adverse reactions that led to discontinuation of DAAs were recorded in our study. Secondly, we have no data regarding viral resistance. Thirdly, we do not have data on patient compliance, as this has not been systematically verified.

\section{CONCLUSIONS}

Our real-life study reported a SVR12 rate of $85.1 \%$ in ITT analysis and of $90.8 \%$ per protocol analysis, in decompensated cirrhosis, comparable with the data resulting from clinical trials and other real-life studies. SVR12 rates did not differ significantly between patients treated for 12 or 24 weeks, and between those treated with or without RBV. The main predictors of SVR were advanced age and lower bilirubin levels. An improvement of their liver function was observed in $66 \%$ of patients after therapy, LDV/SOF being very well tolerated in our difficult-to treat cases, with serious adverse events reported in only $16 / 349$ (4.6\%) of the cases, most of them represented by worsening of the liver function $(9 / 16)$.

Conflicts of interest: L.S.G., L.I., A.T., M.D. received honoraria and educational grants from Gilead. The other authors declare no conflicts of interest concerning this article.

Authors' contributions: L.S.G and M.M. designed the study and critically revised the paper. C.M.P. drafted the manuscript. L.I., A.T., C.S. data acquisition and critical revision of the manuscript. D.I. and C.B statistical analysis. A.E.C., C.S.P., L.T., S.I., C.T., C.M.: data acquisition. M.D., T.V. critically revised the manuscript. All authors read and approved the final manuscript.

Ethics approval statement: The study was approved by the National Ethics Committee of Medicines and Medical Devices (No 27SNI/ OCTOBER 10, 2016).

\section{REFERENCES}

1. World Health Organization. WHO GLOBAL HEPATITIS REPORT, 2017. Available at:https://www.afro.who.int/sites/default/files/201706/9789241565455-eng.pdf. Accessed: 20 Jan 2019.

2. Polaris Observatory HCV Collaborators. Global prevalence and genotype distribution of hepatitis $\mathrm{C}$ virus infection in 2015: a modelling study. Lancet Gastroenterol Hepatol 2017;2:161-176. doi:10.1016/ S2468-1253(16)30181-9

3. Gheorghe L, Csiki IE, Iacob S, Gheorghe C, Smira G, Regep L. The prevalence and risk factors of hepatitis $C$ virus infection in adult population in Romania: a nationwide survey 2006 - 2008. J Gastrointestin Liver Dis 2010;19:373-379.

4. Preda CM, Popescu CP, Baicus C, et al. Real-world efficacy and safety of ombitasvir, paritaprevir/r+dasabuvir+ribavirin in genotype $1 \mathrm{~b}$ patients with hepatitis C virus cirrhosis. Liver Int 2018;38:602-610. doi:10.1111/ liv. 13550

5. Preda CM, Popescu CP, Baicus C, et al. Risk of hepatitis B virus reactivation in hepatitis $\mathrm{B}$ virus + hepatitis $\mathrm{C}$ virus-co-infected patients with compensated liver cirrhosis treated with ombitasvir, paritaprevir/r + dasabuvir + ribavirin. J Viral Hepat 2018;25:834-841. doi:10.1111/jvh.12872

6. Preda CM, Baicus C, Sandra I, et al. Recurrence rate of hepatocellular carcinoma (HCC) in patients with treated HCC and HCV-associated cirrhosis after Ombitasvir, Paritaprevir/r+Dasabuvir+Ribavirin therapy. United European Gastroenterol J. 2019;7:699-708. doi:10.1177/2050640619841254

7. Manuc M, Preda CM, Popescu CP, et al. New epidemiologic data regarding hepatitis $\mathrm{C}$ virus infection in Romania. J Gastrointestin Liver Dis 2017;26:381-386. doi:10.15403/jgld.2014.1121.264.cvr

8. Reddy KR, Bourlière M, Sulkowski M, et al. Ledipasvir and sofosbuvir in patients with genotype 1 hepatitis $C$ virus infection and 
compensated cirrhosis: An integrated safety and efficacy analysis. Hepatology 2015;62:79-86. doi:10.1002/hep.27826

9. Butt AA, Yan P, Shaikh OS, Chung RT, Sherman KE; ERCHIVES study. Sofosbuvir-based regimens in clinical practice achieve SVR rates closer to clinical trials: results from ERCHIVES. Liver Int 2016;36:651-658. doi:10.1111/liv.13036

10. Tapper EB, Bacon BR, Curry MP, et al. Real-world effectiveness for 12 weeks of ledipasvir-sofosbuvir for genotype 1 hepatitis $\mathrm{C}$ : the Trio Health study. J Viral Hepat 2017;24:22-27. doi:10.1111/jvh.12611

11. Ioannou GN, Beste LA, Chang MF, et al. Effectiveness of Sofosbuvir, Ledipasvir/Sofosbuvir, or Paritaprevir/Ritonavir/ Ombitasvir and Dasabuvir Regimens for Treatment of Patients with Hepatitis C in the Veterans Affairs National Health Care System. Gastroenterology 2016;151:457-471.e5. doi:10.1053/j. gastro.2016.05.049

12. Flisiak R, Pogorzelska J, Flisiak-Jackiewicz M. Hepatitis C: efficacy and safety in real life. Liver Int 2017;37 Suppl 1:26-32. doi:10.1111/liv.13293

13. Barone M, Iannone A, Shahini E, et al. A different perspective on sofosbuvir-ledipasvir treatment of patients with HCV genotype 1b cirrhosis: The ital-c network study. J Viral Hepat 2018;25:56-62. doi:10.1111/jvh.12765

14. Lim JK, Liapakis AM, Shiffman ML, et al. Safety and Effectiveness of Ledipasvir and Sofosbuvir, With or Without Ribavirin, in TreatmentExperienced Patients With Genotype 1 Hepatitis C Virus Infection and Cirrhosis. Clin Gastroenterol Hepatol 2018;16:1811-1819.e4. doi:10.1016/j.cgh.2017.12.037

15. Tsuji K, Kurosaki M, Itakura J, et al. Real-world efficacy and safety of ledipasvir and sofosbuvir in patients with hepatitis $\mathrm{C}$ virus genotype 1 infection: a nationwide multicenter study by the Japanese Red Cross Liver Study Group. J Gastroenterol 2018;53:1142-1150. doi:10.1007/ s00535-018-1455-1

16. Charlton M, Everson GT, Flamm SL, et al. Ledipasvir and sofosbuvir plus ribavirin for treatment of $\mathrm{HCV}$ infection in patients with advanced liver disease. Gastroenterology 2015;149:649-659. doi:10.1053/j. gastro.2015.05.010

17. Manns M, Samuel D, Gane EJ, et al. Ledipasvir and sofosbuvir plus ribavirin in patients with genotype 1 or 4 hepatitis $C$ virus infection and advanced liver disease: a multicentre, open-label, randomized, phase 2 trial. Lancet Infect Dis 2016;16:685-697. doi:10.1016/S14733099(16)00052-9

18. Foster GR, Irving WL, Cheung MCM, et al. Impact of direct acting antiviral therapy in patients with chronic hepatitis $\mathrm{C}$ and decompensated cirrhosis. J Hepatol 2016;64:1224-1231. doi:10.1016/j. jhep.2016.01.029
19. Cheung MCM, Walker AJ, Hudson BE, et al. Outcomes after successful direct-acting antiviral therapy for patients with chronic hepatitis $\mathrm{C}$ and decompensated cirrhosis. J Hepatol 2016;65:741-747. doi:10.1016/j. jhep.2016.06.019

20. Guarino M, Morisco F, Valvano MR, et al. Systematic review: interferonfree regimens for patients with $\mathrm{HCV}$-related Child C cirrhosis. Aliment Pharmacol Ther 2017;45:1193-1200. doi:10.1111/apt.14017

21. Idilman R, Demir M, Aladag M, et al. Low recurrence rate of hepatocellular carcinoma following ledipasvir and sofosbuvirtreatment in a real-world chronic hepatitis C patients cohort. J Viral Hepat 2019;26:666-674. doi:10.1111/jvh.13075

22. El-Sherif O, Jiang ZG, Tapper EB, et al. Baseline Factors Associated With Improvements in Decompensated Cirrhosis After Direct-Acting Antiviral Therapy for Hepatitis C Virus Infection. Gastroenterology 2018;154:2111-2121.e8. doi:10.1053/j. gastro.2018.03.022

23. Afdhal N, Reddy KR, Nelson DR, et al. Ledipasvir and sofosbuvir for previously treated HCV genotype 1 infection. N Engl J Med 2014;370:1483-1493. doi:10.1056/NEJMoa1316366

24. Lawitz E, Poordad FF, Pang PS, et al. Sofosbuvir and ledipasvir fixeddose combination with and without ribavirin in treatment-naive and previously treated patients with genotype 1 hepatitis $C$ virus infection (LONESTAR): an open-label, randomised, phase 2 trial. Lancet 2014;383:515-523. doi:10.1016/S0140-6736(13)62121-2

25. Gray E, O’Leary A, Bergin C, et al. Effectiveness of interferon-free therapy for the treatment of HCV-patients with compensated cirrhosis treated through the Irish early access program. Expert Rev Gastroenterol Hepatol 2017;11:593-601. doi:10.1080/17474124.2017.1292850

26. Younossi ZM, Stepanova M, Henry L, et al. Sofosbuvir and ledipasvir are associated with high sustained virologic response and improvement of health-related quality of life in East Asian patients with hepatitis C virus infection. J Viral Hepat 2018;25:1429-1437. doi:10.1111/jvh.12965

27. Terrault NA, Zeuzem S, Di Bisceglie AM, et al. Effectiveness of Ledipasvir-Sofosbuvir Combination in Patients With Hepatitis C Virus Infection and Factors Associated With Sustained Virologic Response. Gastroenterology 2016;151:1131-1140.e5. doi:10.1053/j. gastro.2016.08.004

28. Ekpanyapong S, Reddy KR. Hepatitis C virus therapy in advanced liver disease: Outcomes and challenges. United European Gastroenterol J 2019;7:642-650. doi:10.1177/2050640619840149

29. Backus LI, Belperio PS, Shahoumian TA, Loomis TP, Mole LA. Real world effectiveness of ledipasvir/sofosbuvir in 4365 treatment-naïve, genotype 1 hepatitis C-infected patients. Hepatology 2016;64:405-414 doi:10.1002/hep.28625 